\title{
Identification of Key Candidate Genes and Pathways of Candida albicans-Infected Human Umbilical Vein Endothelial Cells and Drug Screening
}

\author{
Wei Jiang ${ }^{1} \cdot$ Ping Liu $^{2} \cdot$ Jianlei Zhang ${ }^{2} \cdot$ Wenjie Yang'
}

Received: 10 June 2019/ Accepted: 4 December 2019/Published online: 13 December 2019

(C) The Author(s) 2019

\begin{abstract}
Candida albicans is a common opportunistic pathogen that can cause serious infection by blood transmission. C. albicans enters the blood circulation and adheres to the endothelial cells of the vascular wall. However, the detailed mechanism of the effect of $C$. albicans on the endothelial cells remains unclear. In this study, the microarray expression profile of human umbilical vein endothelial cells exposed to $C$. albicans was analyzed. The 191 up-regulated genes were enriched in TNF, T cell receptor, and NF-kappa B signaling pathways. The 71 down-regulated genes were enriched in pyruvate metabolic, purine nucleotide metabolic, purine nucleotide biosynthetic, and humoral immune response processes. Gene set enrichment analysis showed that apoptosis, oxidative phosphorylation, IL6/JAK/STAT3 signaling pathways were enriched. Moreover, two hub genes with a high degree of connectivity, namely, MYC and IL6, were selected. Molecular screening of traditional Chinese medicine libraries was performed on the basis of the structure of MYC protein. The okanin had the highest docking score. MYC might be used as molecular targets for treatment. In addition, okanin may inhibit the infection of $C$. albicans. Thus, MYC can be subjected to further research.
\end{abstract}

Electronic supplementary material The online version of this article (https://doi.org/10.1007/s12088-019-00847-5) contains supplementary material, which is available to authorized users.

Wenjie Yang

explorer@mail.nankai.edu.cn

1 Department of Infectious Diseases, Tianjin First Center Hospital, No. 24 Fukang Road, Nankai District, Tianjin 300192, China

2 Laboratory of Microbiology of Tianjin First Center Hospital, Tianjin, China
Keywords Bioinformatics analysis - Candida albicans . HUVECs · MYC

\section{Introduction}

Candida albicans is a polymorphic yeast and one of the most important pathogens that causes iatrogenic infections in immunodeficient populations [1]. In general, it is a common parasite of humans that can be found in the oropharynx, gastrointestinal tract, and vaginal mucosa and does not cause human diseases. However, when the host's local micro-environment is dysregulated, or the mucosal barrier is impaired, C. albicans can cause mucosal infections, thereby causing diseases such as thrush, fungal vaginitis, and rash $[2,3]$. In some cases, $C$. albicans can invade the mucosal epithelium and vascular endothelium, thereby causing disseminated infections in susceptible populations [4]. In the pathology of the abovementioned diseases, $C$. albicans invades the host's non-phagocytic cells, such as endothelial cells, and plays a vital role in the early stages of the disease [5]. Currently, no mechanism study has been conducted on the effects of $C$. albicans on endothelial cells.

High-throughput virtual screening revealed that okanin has the highest docking score with MYC [6, 7]. Okanin is a chalcone compound found in the genus Bidens [8]. Pharmacological studies have shown that okanin has many effects, such as lowering blood sugar, lowering blood pressure, lowering blood fat, and resisting oxidation [9]. Although the anti-inflammatory activity of okanin has been studied [10], the anti-C. albicans infection activity of okanin has not been investigated yet.

In the present study, differentially expressed genes (DEGs) of endothelial cells after C. albicans infection were 
analyzed by performing bioinformatics analysis. The key functions and pathways of endothelial cells after C. albicans infection were also identified. By conducting the topological analysis, we found and further screened the key hub genes in the affected endothelial cell lesions to obtain okanin. The candidate molecule for traditional Chinese medicine (TCM) may inhibit $C$. albicans infection.

\section{Materials and Methods}

\section{RNA Sequence Analysis}

The RNA expression profiling of GSE7355 downloaded from the GEO database was used in this study. Four independent experiments compared the expression profiles of untreated HUVEC monolayer and of C. albicans-infected HUVEC monolayer. The data quality was assessed by calculating residuals.sign, residuals, weight, relative log expression (RLE), normalized unscaled standard errors (NUSE), and RNA degradation. We used the R packages of pheatmap and limma to analyze the difference in RNA expression profiling among the eight groups. We set the llog2fold changel $\geq 0.6$ of the cutoff limit for DEGs.

\section{Gene Set Enrichment Analysis (GSEA)}

GSEA was conducted using the GSEA software [11].

\section{Omics Analysis}

The DEGs were further analyzed using the gene ontology (GO), KEGG pathway, and protein-protein interaction (PPI) analyses [12]. The GO and pathway analysis were conducted using the website of Metascape (http://metas cape.org). The enriched pathways or functions were then drawn into bubble maps with the R package of ggplot2. PPI annotation of the DEGs was retrieved from the STRING database. The PPI network was visualized using the Cytoscape software. Then, the Cytoscape apps of CentiScape and MCODE were used for detecting densely connected regions $[13,14]$.

\section{TCM Database and Protein Preparation}

A total of 32,364 TCM molecules were obtained from the TCM database (http://tcm.cmu.edu.tw/) [15]. All the TCM molecules were refined by removing the counterions and salts and adding hydrogen atoms. Then, we performed energy minimization using Schrodinger software [16, 17]. For protein preparation, the crystal structure of MYC was downloaded from the protein data bank (http://www.rcsb. org/, PDBID: 1NKP) [18]. The protein structure was refined by removing crystalline water and ions. Then, we added hydrogen atoms and performed energy minimization of the protein structure. We selected high-throughput virtual screening model of Schrodinger to perform molecular docking. Glide XP (extra precision) was used for the final 10 TCM molecules calculations [19, 20].

\section{Luciferase Report Assay}

The HUVEC cell lines, obtained from KeyGen Biotech (Nanjing, China), were transfected with dual-reporter constructs of MYC using the transfection regents. After transfection of $24 \mathrm{~h}$, cells were treated with $40 \mu \mathrm{M}$ of okanin. After another $48 \mathrm{~h}$, the culture medium was collected into a 96-well plate and the luminescence was measured using a luminometer. The fluorescence intensity reflects the transcriptional activity.

\section{Western Blot Assay}

The okanin was added to the HUVEC cell culture at a concentration of $40 \mu \mathrm{M}$ when the cell density reached to $80 \%$. After incubation of $48 \mathrm{~h}$, the cells were lysed and 30 $\mu \mathrm{g}$ of total protein was used for sodium dodecyl sulfatepolyacrylamide gel electrophoresis and transferred onto polyvinylidene difluoride membranes (Millipore, USA). The membrane was incubated with primary antibodies against GLUT2 (1:1000, Affinity Bioreagents, USA), MYC (1:1000, Affinity Bioreagents, USA), and GAPDH (1:5000, Affinity Bioreagents, USA), followed by the secondary antibody (1:5000, Affinity Bioreagents, USA). The blots were detected using an enhanced chemiluminescence detection kit (Millipore, USA). The expression analysis was performed using the ImageJ software. The ratio of densitometry value to the corresponding GAPDH was used to reflect the relative protein expression.

\section{Results \\ C. albicans Affected the RNA Expression Profile in HUVEC Cell Lines}

To clarify the effects of $C$. albicans on endothelial cells, we downloaded the GEO database of GSE7355 from the GEO database. By calculating residuals.sign, residuals, weight, RLE, NUSE, and RNA degradation, the data quality was acceptable (Figure S1A-F). The heat map analysis result showed a quite different expressed gene profile between the control HUVEC monolayer and C. albicans-infected HUVEC monolayer (Figure S2). The volcano plot further described a difference in gene expression profile between the two groups. For instance, many genes were 
considerably upregulated and labeled in red color, whereas some genes were remarkably downregulated and labeled in blue color (Figure S3).

\section{C. albicans Infection Affected Apoptosis, Metabolism, and Inflammation Related Pathways}

The omics analysis was then conducted with the screened DEGs. The GSEA analysis results showed that $C$. albicans infection could affect the apoptosis and oxidative phosphorylation processes. Furthermore, the $C$. albicans infection could affect the inflammatory response and its related signaling pathways, namely, NFKB, IL6-JAK-STAT3, and IL2-STAT5 pathways (Figure S4). Further analysis of the DEGs revealed that $C$. albicans infection extensively promoted immune- and inflammation-related signaling pathways, such as TNF, Toll-like receptor, T cell receptor, NFkappa B, and chemokine-mediated signaling pathway (Fig. 1a). The metabolism-related pathways, such as glycolytic and carbohydrate catabolic processes, were almost completely suppressed by $C$. albicans infection, thereby inhibiting synthesis of nicotinamide nucleotide and ATP (Fig. 1b). Multiple biological processes and pathways were implicated in HUVEC monolayer by $C$. albicans infection. On this basis, we constructed $C$. albicans-related network using PPI information of DEGs (Supplemental table). This network consisted of 105 nodes and 360 edges (Fig. 2a). By using the CentiScape and MCODE APPs, we identified the topological network with features of Degree and K-Core. Three hub networks were screened with the network scoring of $6.737,6$, and 3, respectively (Fig. 2b). As a result, some potential hub nodes, such as MYC, IL6, FOS, and NFKBIA, were identified as candidate targets for treating C. albicans infection (Table 1).

\section{Candidate Drugs Screening for Treating $C$. albicans Infection}

In accordance with the omics analysis, MYC was detected to be a hub node and an important gene related to inflammation and transformation of inflammatory cancer. Therefore, we chose the gene MYC for drug screening. A systematic strategy for identifying TCM molecules were designed by using structure-based VS. The high-throughput virtual screening process is shown in Fig. 3a. HTV screen method yielded 100 TCM molecules with the highest MYC score. Furthermore, twenty TCM molecules were further screened out form the previous $100 \mathrm{TCM}$ molecules. Finally, 10 TCM molecules were obtained (Table 2). We performed extra precision calculation with MYC-DNA complex. Among these molecules, okanin had the highest docking score. The interactions between MYC-DNA and okanin are shown in Fig. 3b. Okanin interacted with key amino acid residues (Arg-239 and Arg-914) in the DNA binding site of MYC via two hydrogen bonding interactions. In DNA helix, okanin formed hydrogen bond interaction with three bases. Okanin could inhibit the binding sites of MYC and DNA, thereby inhibiting the transcriptional activity of MYC. To further demonstrate the activity of okanin on treatment of C. albicans infection, the luciferase assay of MYC and MYC downstream protein expression of GLUT2 and p65 were conducted [21, 22]. The luciferase assay results showed that okanin could significantly inhibit the transcriptional regulatory activity of MYC (Fig. 3c). Meanwhile, the expressions of MYC downstream proteins of GLUT2 and p65 were also significantly inhibited by okanin (Fig. 3d).

\section{Discussion}

Microorganisms in the lumen enter the parenchymal tissue through their interaction with endothelium and then cause infection by increasing the vascular permeability [23]. This occurrence is the first and key step of disseminated infections [24]. Hence, vascular endothelial cells play a key role in the early stage of hematogenous disseminated infections and is a tool for studying fungal infections [25]. Thus, studying the effect of $C$. albicans on vascular endothelial cells can clarify the pathogenesis of disseminated candidiasis. Although there have be some treatments for $C$. albicans [26], more effective treatment methods need to be selected and developed. C. albicans invades human host cells and is associated with cell motility, cytoskeleton actin, PI3K signaling pathway, and membrane receptors EGFR and Erbb2 [27-30]. From a microbial point of view, the activity of pathogenic fungi, mycelial growth, and the virulence factors of $C$. albicans, such as als3 and Ssa1, all play an important role in the whole process [31].

Hub genes play vital roles during the progression of $C$. albicans infection. Although many studies on C. albicansinfected endothelial cells are available, much effort is still needed to identify hub genes and develop candidate drugs that may inhibit $C$. albicans infection. Pathway enrichment analysis indicated that TNF signaling pathway, $\mathrm{T}$ cell receptor signaling pathway, response to chemokine, positive regulation of apoptotic process, and inflammatory response pathways were overrepresented among the upregulated genes.

The PPI network was constructed with 162 nodes/DEGs. The PPI network and the topological index revealed that 
A

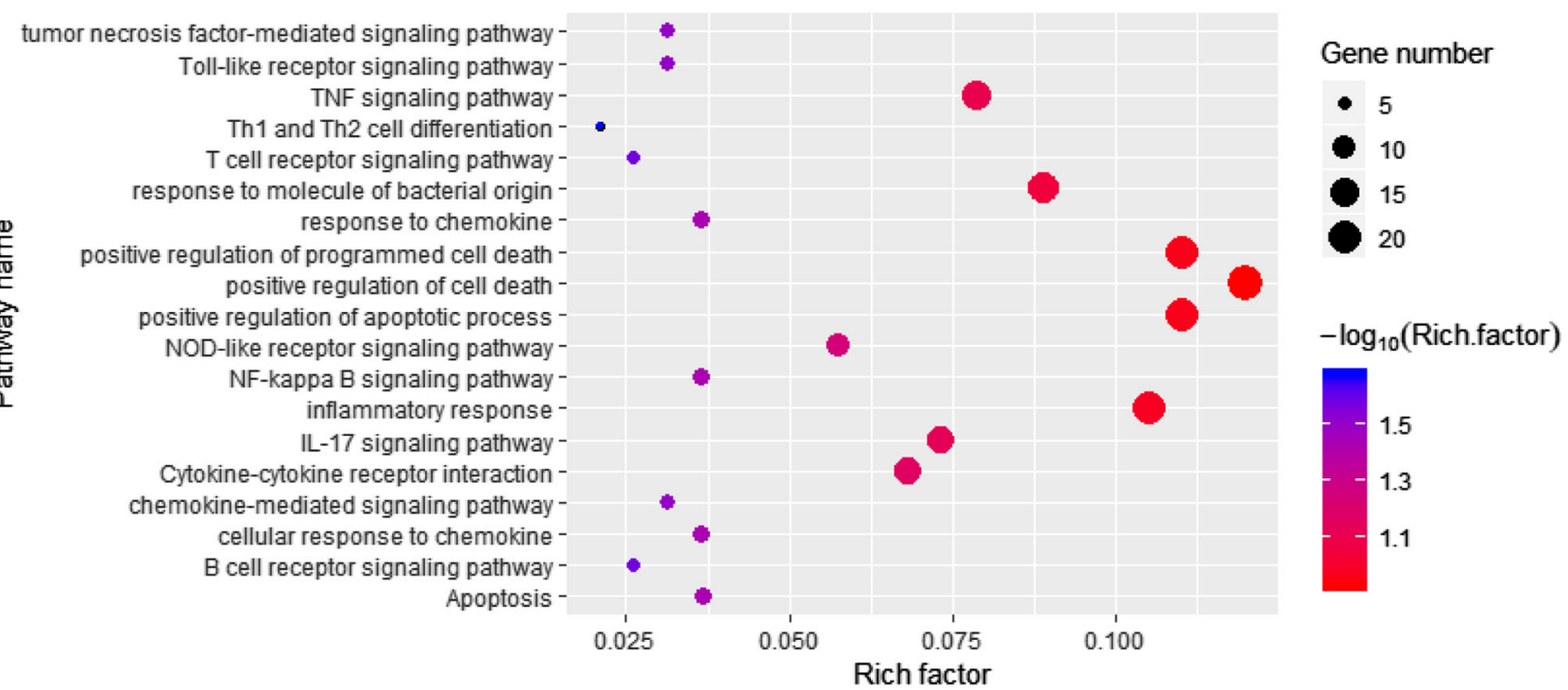

B

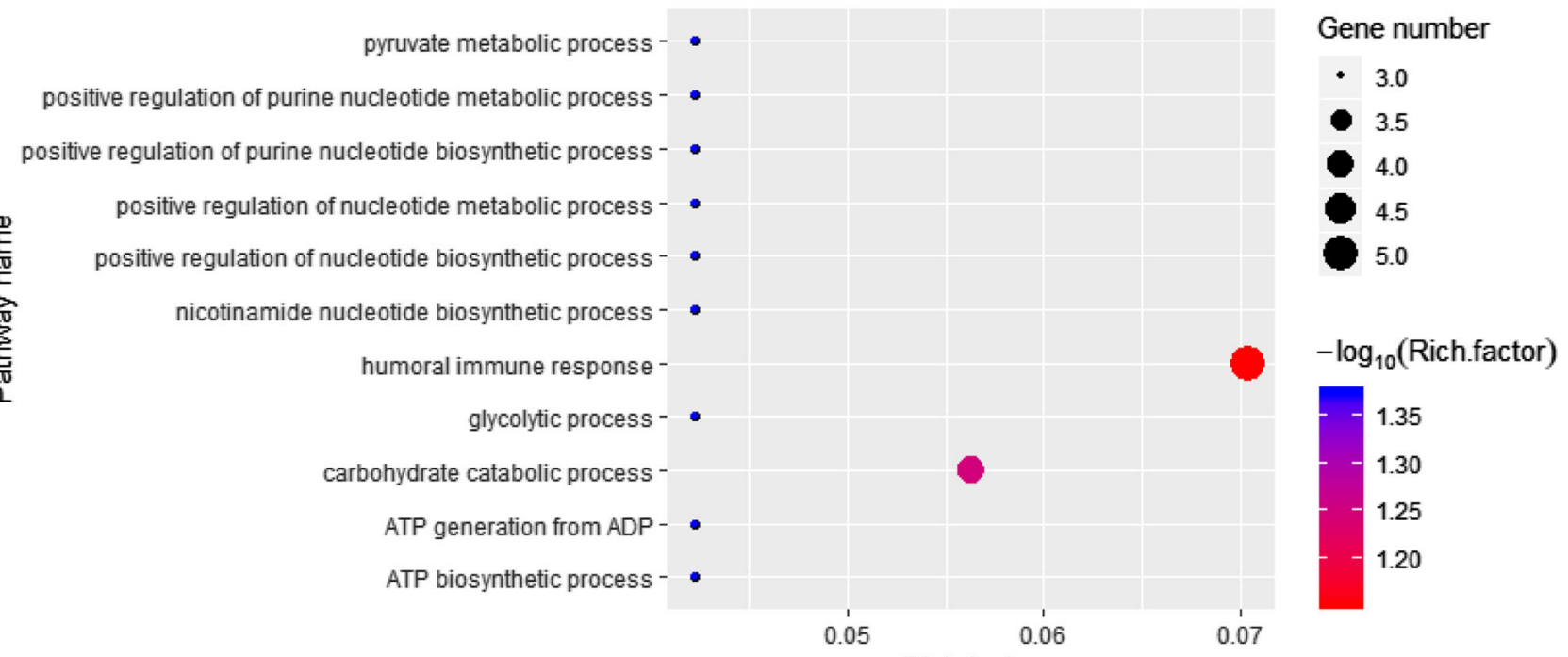

Fig. 1 Effects of Candida albicans infection on biological processes and pathways in HUVEC cells. a Pathway analysis of the upregulated genes by $C$. albicans infection revealed an activation of immune and inflammation related pathways including $\mathrm{TNF}$, Toll-like receptor, $\mathrm{T}$ cell receptor, NF-kappa $\mathrm{B}$, and chemokine-mediated signaling

IL6, FOS, and MYC were the most important in this PPI network. These proteins have a high degree of expression and a central regulatory role in the PPI network. Hence, they are likely to be regulatory hubs.

MYC, a transcription factor, can bind DNA in a nonspecific manner [32]. MYC gene is an important member of MYC gene family. It is not only a translocation gene but also an adjustable gene regulated by many substances. It can make cells proliferate indefinitely and obtain the function of immortalization; it promotes cell division $[33,34]$. It is also involved in cell apoptosis and is related pathway. b Pathway analysis of the downregulated genes by $C$. albicans infection revealed an inhibition on metabolism related processes like synthesis of nicotinamide nucleotide and ATP (false discovery rate $<0.05, \log 2$ fold changel $\geq 0.6$ )

to the development of various tumors [35]. In this study, we used the crystal structure of MYC-DNA complex to screen TCM molecules that may inhibit MYC activity. By multi-level virtual screening, 10 TCM molecules with potential inhibitory activity were obtained; among these molecules, okanin had the highest docking score.

This study demonstrated that okanin, a major effective constituent of Bidens (Asteraceae), may inhibit C. albicans infection by inhibiting MYC. Okanin can lower the mRNA and protein level of IL6 in the LPS-induced pro-inflammatory model [10]. It also potently inhibited a number of 
Fig. 2 PPI network of DEGs. a PPI analysis of the upregulated genes by Candida albicans infection by Cytoscape software, where the node size reflected the gene interaction degree, larger circles indicated higher gene interaction degree. b Seed nodes analysis of the PPI network by MCODE. Three modules identified by MCODE showed the candidate targets of treating $C$. albicans infection, with the network scoring of $6.737,6$, and 3 .

Table 1 Top hub proteins in the PPI network based on interaction degree
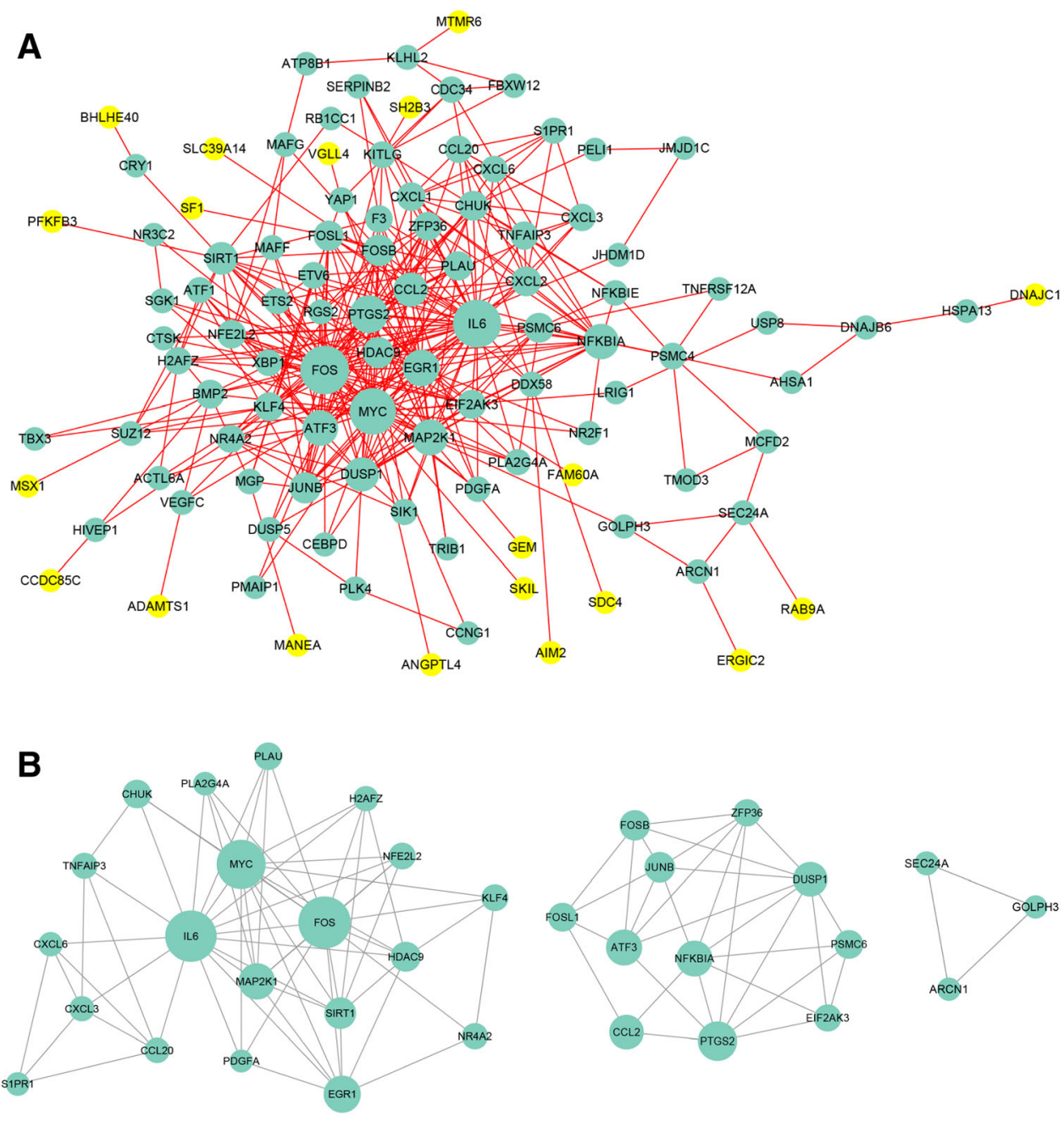

\begin{tabular}{llcllc}
\hline Gene name & Degree unDir & Bridging unDir & Eigen vector unDir & Radiality unDir & Stress unDir \\
\hline IL6 & 47 & 13.838 & 0.334 & 6.683 & 15,962 \\
FOS & 43 & 9.326 & 0.315 & 6.602 & 11,290 \\
MYC & 40 & 13.342 & 0.282 & 6.621 & 14,748 \\
PTGS2 & 27 & 15.575 & 0.248 & 6.491 & 5250 \\
MAP2K1 & 24 & 12.665 & 0.197 & 6.385 & 5532 \\
EGR1 & 23 & 10.907 & 0.215 & 6.416 & 3646 \\
CCL2 & 23 & 12.011 & 0.201 & 6.329 & 3150 \\
NFKBIA & 21 & 38.857 & 0.195 & 6.422 & 7938 \\
ATF3 & 21 & 6.814 & 0.185 & 6.298 & 1976 \\
DUSP1 & 19 & 17.998 & 0.184 & 6.323 & 2984 \\
\hline
\end{tabular}

pro-inflammatory responses in cells [8]. The structure of okanin contained $\alpha-\beta$ unsaturated carbonyl moiety that was composed of two hydroxyphenyl rings and a three-carbon unit. The binding model of okanin and MYC-DNA complex revealed that okanin was in the middle of MYC binding to DNA and inhibited the transcriptional activity of MYC. Okanin interacted with Arg239 and Arg914 of MYC to form two hydrogen bonds. Okanin also interacted with DA109, DC110, and DC310 on DNA [18]. Therefore, blocking interaction of MYC and DNA was observed. Okanin inhibited MYC transcriptional activity, possibly also inhibiting the transformation of inflammatory cancer caused by $C$. albicans infection. 
A

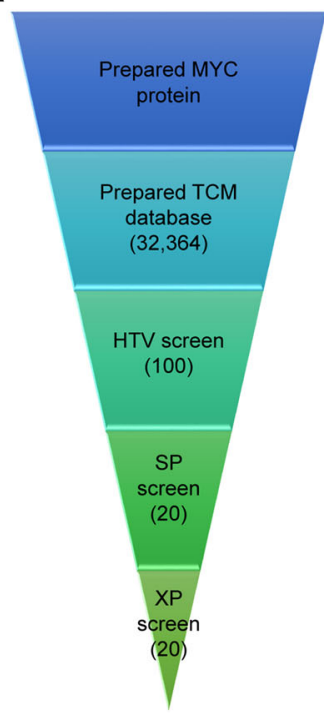

C

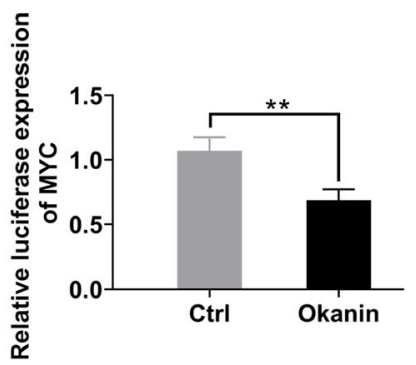

B

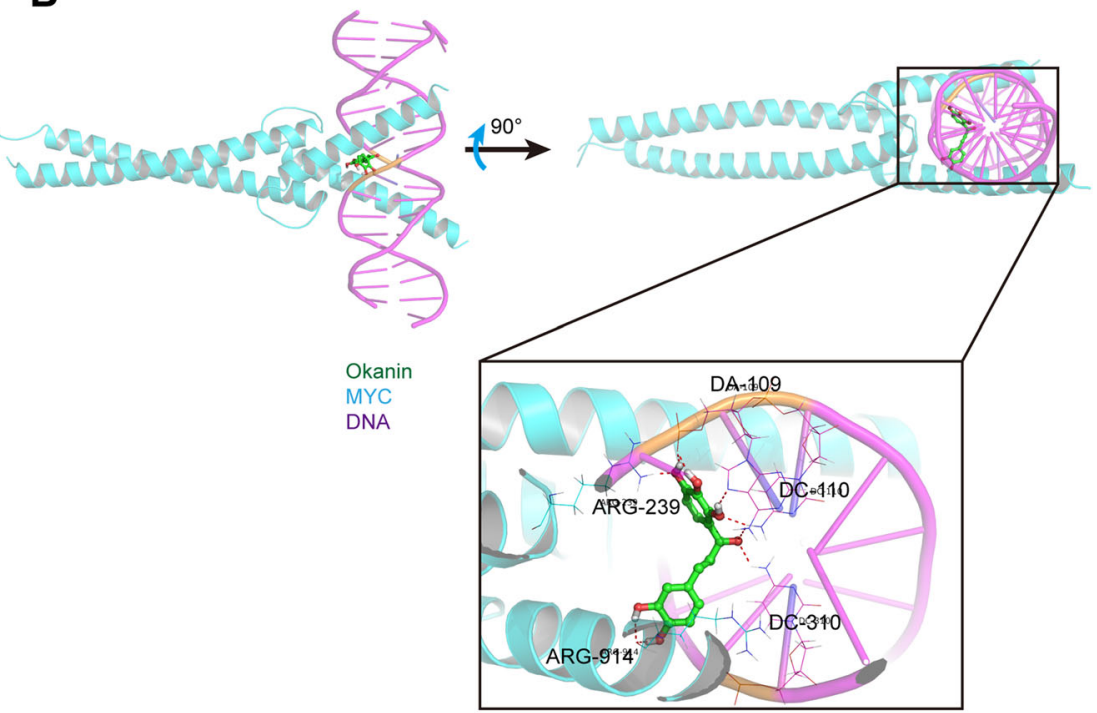

D
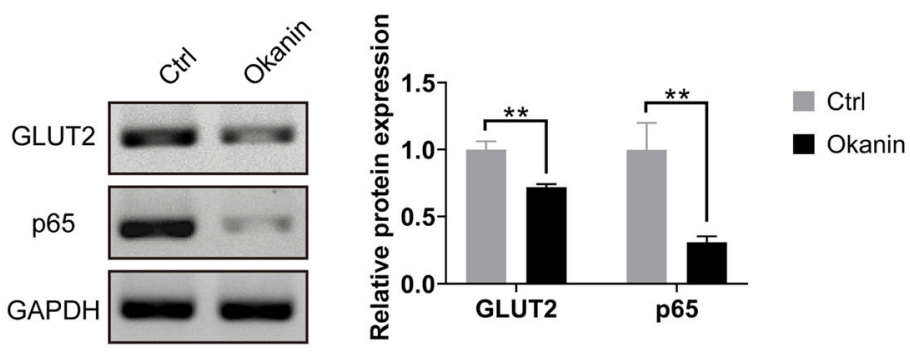

Fig. 3 Receptor-ligand interactions of compound. a Protocol flowchart of MYC inhibitor discovery strategy, ten candidate inhibitors including okanin were screened at last through HTV, SP, and XP screen methods. b Binding model of MYC-DNA complexes with okanin (PDB code: 1NKP) through molecular docking method. The results showed that okanin interacted with two key amino acid residues in the DNA binding site of MYC. c Okanin could significantly inhibit the transcriptional regulatory activity of MYC by luciferase assay. d Okanin significantly inhibited the expressions of GLUT2 and p65, which were the downstream proteins of MYC $(\mathrm{n}=3, * * P<0.01)$

Table 2 Detailed docking score of top ten screened TCMs

\begin{tabular}{llccccc}
\hline Compound name & Docking score & Glide evdw & Glide ecoul & Glide energy & Glide einternal & Glide emodel \\
\hline Okanin & -9.97 & 2.14 & -30.85 & -28.71 & 2.52 & -46.83 \\
Prolithospermic acid & -9.38 & -25.15 & -24.53 & -49.67 & 7.15 & -50.26 \\
Eximine & -8.91 & -24.27 & -18 & -42.27 & 12.27 & -60.42 \\
Chalconaringenin & -8.32 & -18.09 & -21.36 & -39.44 & 3.33 & -48.89 \\
Maclurin & -8.27 & -21.93 & -20.63 & -42.57 & 2.57 & -56.93 \\
(+)-Catechin & -6.93 & -23.54 & -12.38 & -35.92 & 2.23 & -48.89 \\
Licochalcone B & -6.93 & -15.63 & -14.66 & -30.29 & 7.53 & -23.07 \\
Pinobaksin & -6.63 & -20.57 & -7.66 & -28.23 & 0.14 & -44.29 \\
Citreorosein & -6.48 & -26.89 & -21.76 & -48.65 & 1.95 & -63.36 \\
2'-Methoxy-3,4,4'-trihydroxychalcone & -6.35 & -20.75 & -17.07 & -37.82 & 6.79 & -49.1 \\
\hline
\end{tabular}


Acknowledgements This study was supported by the Science and Technology Fund of Tianjin health and Family Planning Commission [Grant 2012KZ022].

\section{Compliance with Ethical Standards}

Conflict of interest The authors declare no competing interests

Open Access This article is licensed under a Creative Commons Attribution 4.0 International License, which permits use, sharing, adaptation, distribution and reproduction in any medium or format, as long as you give appropriate credit to the original author(s) and the source, provide a link to the Creative Commons licence, and indicate if changes were made. The images or other third party material in this article are included in the article's Creative Commons licence, unless indicated otherwise in a credit line to the material. If material is not included in the article's Creative Commons licence and your intended use is not permitted by statutory regulation or exceeds the permitted use, you will need to obtain permission directly from the copyright holder. To view a copy of this licence, visit http://creativecommons. org/licenses/by/4.0/.

\section{References}

1. Pujol C, Reynes J, Renaud F, Raymond M, Tibayrenc M, Ayala FJ, Janbon F, Mallie M, Bastide JM (1993) The yeast Candida albicans has a clonal mode of reproduction in a population of infected human immunodeficiency virus-positive patients. Proc Natl Acad Sci USA 90:9456-9459

2. Morales DK, Hogan DA (2010) Candida albicans interactions with bacteria in the context of human health and disease. PLoS Pathog 6:e1000886. https://doi.org/10.1371/journal.ppat.1000886

3. Nobile CJ, Johnson AD (2015) Candida albicans biofilms and human disease. Annu Rev Microbiol 69:71-92. https://doi.org/10. 1146/annurev-micro-091014-104330

4. Hostetter MK (1994) Adhesins and ligands involved in the interaction of Candida spp. with epithelial and endothelial surfaces. Clin Microbiol Rev 7:29-42

5. Filler SG, Swerdloff JN, Hobbs C, Luckett PM (1995) Penetration and damage of endothelial cells by Candida albicans. Infect Immun 63:976-983

6. Arya H, Coumar MS (2014) Virtual screening of traditional Chinese medicine (TCM) database: identification of fragmentlike lead molecules for filariasis target asparaginyl-tRNA synthetase. J Mol Model 20:2266. https://doi.org/10.1007/s00894014-2266-9

7. Wang W, Wan M, Liao D, Peng G, Xu X, Yin W, Guo G, Jiang F, Zhong W, He J (2017) Identification of potent chloride intracellular channel protein 1 inhibitors from traditional Chinese medicine through structure-based virtual screening and molecular dynamics analysis. Biomed Res Int 2017:4751780. https://doi. org/10.1155/2017/4751780

8. Kil JS, Son Y, Cheong YK, Kim NH, Jeong HJ, Kwon JW, Lee EJ, Kwon TO, Chung HT, Pae HO (2012) Okanin, a chalcone found in the genus Bidens, and 3-penten-2-one inhibit inducible nitric oxide synthase expression via heme oxygenase-1 induction in RAW264.7 macrophages activated with lipopolysaccharide. J Clin Biochem Nutr 50:53-58. https://doi.org/10.3164/jcbn.1130

9. Patten GS, Abeywardena MY, Bennett LE (2016) Inhibition of angiotensin converting enzyme, angiotensin II receptor blocking, and blood pressure lowering bioactivity across plant families. Crit
Rev Food Sci Nutr 56:181-214. https://doi.org/10.1080/ 10408398.2011.651176

10. Hou Y, Li G, Wang J, Pan Y, Jiao K, Du J, Chen R, Wang B, Li N (2017) Okanin, effective constituent of the flower tea Coreopsis tinctoria, attenuates LPS-induced microglial activation through inhibition of the TLR4/NF-kappaB signaling pathways. Sci Rep 7:45705. https://doi.org/10.1038/srep45705

11. Subramanian A, Kuehn H, Gould J, Tamayo P, Mesirov JP (2007) GSEA-P: a desktop application for gene set enrichment analysis. Bioinformatics 23:3251-3253. https://doi.org/10.1093/ bioinformatics/btm369

12. Szklarczyk D, Franceschini A, Wyder S, Forslund K, Heller D, Huerta-Cepas J, Simonovic M, Roth A, Santos A, Tsafou KP, Kuhn M, Bork P, Jensen LJ, von Mering C (2015) STRING v10: protein-protein interaction networks, integrated over the tree of life. Nucleic Acids Res 43:D447-D452. https://doi.org/10.1093/ nar/gku1003

13. Jiang H, Ma R, Zou S, Wang Y, Li Z, Li W (2017) Reconstruction and analysis of the lncRNA-miRNA-mRNA network based on competitive endogenous RNA reveal functional lncRNAs in rheumatoid arthritis. Mol Biosyst 13:1182-1192. https://doi.org/10.1039/c7mb00094d

14. Bader GD, Hogue CW (2003) An automated method for finding molecular complexes in large protein interaction networks. BMC Bioinform 4:2

15. Sanderson K (2011) Databases aim to bridge the East-West divide of drug discovery. Nat Med 17:1531. https://doi.org/10. 1038/nm1211-1531a

16. Halgren TA, Murphy RB, Friesner RA, Beard HS, Frye LL, Pollard WT, Banks JL (2004) Glide: a new approach for rapid, accurate docking and scoring. 2. Enrichment factors in database screening. J Med Chem 47:1750-1759. https://doi.org/10.1021/ jm030644s

17. Friesner RA, Banks JL, Murphy RB, Halgren TA, Klicic JJ, Mainz DT, Repasky MP, Knoll EH, Shelley M, Perry JK, Shaw DE, Francis P, Shenkin PS (2004) Glide: a new approach for rapid, accurate docking and scoring. 1. Method and assessment of docking accuracy. J Med Chem 47:1739-1749. https://doi.org/10. 1021/jm0306430

18. Nair SK, Burley SK (2003) X-ray structures of Myc-Max and Mad-Max recognizing DNA. Molecular bases of regulation by proto-oncogenic transcription factors. Cell 112:193-205

19. Gupta S, Bajaj AV (2018) Extra precision glide docking, free energy calculation and molecular dynamics studies of 1,2-diarylethane derivatives as potent urease inhibitors. J Mol Model 24:261. https://doi.org/10.1007/s00894-018-3787-4

20. Friesner RA, Murphy RB, Repasky MP, Frye LL, Greenwood JR, Halgren TA, Sanschagrin PC, Mainz DT (2006) Extra precision glide: docking and scoring incorporating a model of hydrophobic enclosure for protein-ligand complexes. J Med Chem 49:6177-6196. https://doi.org/10.1021/jm051256o

21. Osthus RC, Shim H, Kim S, Li Q, Reddy R, Mukherjee M, Xu Y, Wonsey D, Lee LA, Dang CV (2000) Deregulation of glucose transporter 1 and glycolytic gene expression by c-Myc. J Biol Chem 275:21797-21800. https://doi.org/10.1074/jbc. C000023200

22. Srivastava J, Siddiq A, Gredler R, Shen XN, Rajasekaran D, Robertson CL, Subler MA, Windle JJ, Dumur CI, Mukhopadhyay ND, Garcia D, Lai Z, Chen Y, Balaji U, Fisher PB, Sarkar D (2015) Astrocyte elevated gene-1 and c-Myc cooperate to promote hepatocarcinogenesis in mice. Hepatology 61:915-929. https://doi.org/10.1002/hep.27339

23. Debreczeni ML, Nemeth Z, Kajdacsi E, Schwaner E, Mako V, Masszi A, Doleschall Z, Rigo J, Walter FR, Deli MA, Pal G, Dobo J, Gal P, Cervenak L (2019) MASP-1 increases endothelial 
permeability. Front Immunol 10:991. https://doi.org/10.3389/ fimmu.2019.00991

24. Beilke MA (1989) Vascular endothelium in immunology and infectious disease. Rev Infect Dis 11:273-283

25. Neves GW, Curty N, Kubitschek-Barreira PH, Fontaine T, Souza GH, Cunha ML, Goldman GH, Beauvais A, Latge JP, LopesBezerra LM (2016) Dataset of differentially regulated proteins in HUVECs challenged with wild type and UGM1 mutant Aspergillus fumigatus strains. Data Brief 9:24-31. https://doi.org/10. 1016/j.dib.2016.07.062

26. Alqarihi A, Singh S, Edwards JE Jr, Ibrahim AS, Uppuluri P (2019) NDV-3A vaccination prevents $C$. albicans colonization of jugular vein catheters in mice. Sci Rep 9:6194. https://doi.org/10. 1038/s41598-019-42517-y

27. Rollenhagen C, Wollert T, Langford GM, Sundstrom P (2009) Stimulation of cell motility and expression of late markers of differentiation in human oral keratinocytes by Candida albicans. Cell Microbiol 11:946-966. https://doi.org/10.1111/j.1462-5822. 2009.01303.x

28. Akashi T, Kanbe T, Tanaka K (1994) The role of the cytoskeleton in the polarized growth of the germ tube in Candida albicans. Microbiology 140:271-280. https://doi.org/10.1099/13500872140-2-271

29. Moyes DL, Shen C, Murciano C, Runglall M, Richardson JP, Arno M, Aldecoa-Otalora E, Naglik JR (2014) Protection against epithelial damage during Candida albicans infection is mediated by PI3K/Akt and mammalian target of rapamycin signaling. J Infect Dis 209:1816-1826. https://doi.org/10.1093/infdis/jit824
30. Zhu W, Phan QT, Boontheung P, Solis NV, Loo JA, Filler SG (2012) EGFR and HER2 receptor kinase signaling mediate epithelial cell invasion by Candida albicans during oropharyngeal infection. Proc Natl Acad Sci USA 109:14194-14199. https://doi.org/10.1073/pnas.1117676109

31. Sun JN, Solis NV, Phan QT, Bajwa JS, Kashleva H, Thompson A, Liu Y, Dongari-Bagtzoglou A, Edgerton M, Filler SG (2010) Host cell invasion and virulence mediated by Candida albicans Ssa1. PLoS Pathog 6:e1001181. https://doi.org/10.1371/journal. ppat. 1001181

32. Zeller KI, Jegga AG, Aronow BJ, O’Donnell KA, Dang CV (2003) An integrated database of genes responsive to the Myc oncogenic transcription factor: identification of direct genomic targets. Genome Biol 4:R69. https://doi.org/10.1186/gb-2003-410-r69

33. Gordan JD, Thompson CB, Simon MC (2007) HIF and c-Myc: sibling rivals for control of cancer cell metabolism and proliferation. Cancer Cell 12:108-113. https://doi.org/10.1016/j.ccr. 2007.07.006

34. Schuhmacher M, Staege MS, Pajic A, Polack A, Weidle UH, Bornkamm GW, Eick D, Kohlhuber F (1999) Control of cell growth by c-Myc in the absence of cell division. Curr Biol 9:1255-1258

35. Hermeking H, Eick D (1994) Mediation of c-Myc-induced apoptosis by p53. Science 265:2091-2093

Publisher's Note Springer Nature remains neutral with regard to jurisdictional claims in published maps and institutional affiliations. 